\title{
MINERÍA EN AMÉRICA LATINA Y EL CARIBE, UN ENFOQUE SOCIOAMBIENTAL
}

\section{MINING IN LATIN AMERICA AND THE CARIBBEAN, A SOCIOENVIRONMENTAL APPROACH}

\author{
Ricardo Viana Ríos ${ }^{1}$ \\ ${ }^{1}$ Ing. Geólogo, Esp. Gestión Social y Ambiental, M.Sc. Planeación Urbana y Regional, Subdirector Nacional de Minería, Uni- \\ dad de Planeación Minero Energética, Av. Calle 26 No. 69 D-91 Torre 1, Piso 9 Bogotá, D.C., Colombia, e-mail: rvianarios@ \\ gmail.com, (iDhttps://orcid.org/0000-0002-4692-1353
}

Rev. U.D.C.A Act. \& Div. Cient. 21(2):617-637, Julio-Diciembre, 2018 https://doi.org/10.31910/rudca.v21.n2.2018.1066

Artículo de acceso abierto publicado por Revista U.D.C.A Actualidad \& Divulgación Científica bajo

una licencia Creative Commons CC BY-NC 4.0

\section{RESUMEN}

La minería sigue siendo necesaria para el desarrollo de la sociedad e influyente en la economía de los países de América Latina y el Caribe, pero las políticas públicas no son coherentes para mejorar su manejo socioambiental. Por ello, esta revisión tiene por objetivo analizar la incidencia de la minería en la economía de los países de América Latina y el Caribe, con especial enfoque en los aspectos socioambientales, incluidos en los planes de desarrollo nacional. El método de trabajo consistió en una revisión y análisis de la información sobre el tema, contenida en los principales observatorios de la región. Los resultados, se sintetizan en tablas y mapas que expresan: la relación entre la importancia en la economía y potencial minero; el número y distribución de conflictos socioambientales derivados de actividades mineras y, algunos efectos económicos, que dichos conflictos tienen sobre los proyectos mineros.

Palabras clave: industria minera, socioambiental, política pública, política minera, América Latina y el Caribe.

\section{ABSTRACT}

Mining is still necessary for the development of society, and influential in the economy of the countries of Latin America and the Caribbean, but public policies are not coherent to improve their socioenvironmental management. Therefore, this review aims to analyze the impact of mining on the economy of the countries of Latin America and the Caribbean, with a special focus on socio-environmental aspects, included in the national development plans. The working method consisted of a review and analysis of information on the subject, contained in the main observatories of the region. The results are synthesized in tables and maps that express: the relationship between the importance in the economy and mining potential; the number and distribution of socioenvironmental conflicts derived from mining activities; and, some economic effects that such conflicts have on in mining projects.

Keywords: mining industry, socio-environmental, public policy, mining policy, Latin America and the Caribbean.

\section{INTRODUCCIÓN}

La minería, los minerales y los metales son importantes para el desarrollo económico y social de muchos países y los minerales siguen siendo esenciales para la vida moderna (United Nations, 2002). Durante muchos años, se ha destacado la relevancia del sector minero en los diferentes países, desde el punto de vista económico; sin embargo, en las circunstancias actuales, los aspectos socioambientales, toman tanta fuerza que, de no abordarlos adecuadamente, se acumulan conflictos en el desarrollo del sector minero y en la economía de los países de América Latina y el Caribe -ALC-. Por esta razón, resulta importante hacer una revisión sobre la incidencia de la minería en la región; la forma en que los países han estado abordando los aspectos socioambientales asociados con la minería, desde sus planes de desarrollo nacional y los conflictos socioambientales, relacionados con las actividades mineras y sus efectos económicos, medidos en términos monetarios. 
En contexto espacial, las condiciones geológicas de ALC favorecieron la formación de una gran variedad de depósitos minerales con reservas significativas (BID, 2016), lo cual, da lugar a que la explotación minera sea una constante en el abastecimiento de materias primas para el desarrollo de la infraestructura vial, de vivienda y de la industria local. De ahí, la extracción a gran escala de minerales, como cobre, oro, carbón, níquel, que son comercializados en los mercados internacionales, generando inversión extranjera, regalías, impuestos y empleo, para dinamizar la economía de los países de la región (CEPAL, 2013a).

Para la CEPAL (2013b), en ALC, la minería es una actividad que incide en la economía regional y es un importante motor económico para las comunidades en términos de aporte al producto interno bruto -PIB- (CEPAL, 2010); sin embargo, su aprovechamiento se debe hacer respetando el entorno ambiental e identificando los posibles conflictos que genere en el territorio (Viana et al. 1999). Además, los conflictos de carácter ambiental y social están afectando, en diferente grado, el desarrollo del sector minero (United Nations, 2012), influyendo en la inversión en exploración, la puesta en operación de algunos proyectos e, incluso, ha ocasionado la suspensión de grandes proyectos, en etapa de exploración y explotación, con amplias expectativas en la producción de minerales, como oro, carbón y cobre (Jenkins \& Yakovleva, 2006). Como señalan Rodríguez \& Correa (2003), hoy más que nunca, los conflictos socioambientales son una parte central de los procesos de desarrollo y de rearticulación de ACL, en las agendas globales.

En este sentido, los aspectos ambientales se han abordado de manera separada a los sociales, pero es importante considerar que hacen parte de una relación recíproca, necesaria de ser tratada de manera conjunta, ya sea como una oportunidad o un problema, dependiendo de la óptica, el enfoque o el caso específico.

Es por ello que, Leff (2009) hace un llamado a la necesidad de actuar en función de una racionalidad, en la cual, la sociedad y el ambiente funcionen como un todo. Para Espluga \& Lemkow (2017), el pensamiento socioambiental ha sido recurrente en nuestra sociedad y ha adoptado diferentes formas a lo largo de la historia. En concreto, lo socioambiental según Floriani (2014), hace referencia a un paradigma en tránsito, que requiere un sistema de cooperación innovador entre las partes, buscando saberes, metodologías, formas de interpretar, de evaluar y de dar respuesta a diversos temas de nuevas realidades, que corresponden a los fenómenos sociales y ambientales interconectados. En general, desde la óptica de las comunidades, en la actualidad, la minería no es vista como un factor de desarrollo en sus territorios, por el contrario, consideran que es una actividad que contamina al recurso hídrico, genera emisiones a la atmósfera y produ- ce efectos negativos sobre la salud, con una incidencia muy baja en el desarrollo económico de ellas (Banco Mundial, 2012).

Según la CEPAL (2015a), siendo la minería uno de los sectores que en ALC atrae más inversión extranjera directa, también es una de las actividades que mayores impactos produce sobre el ambiente, generando cinco veces más de emisiones de gases efecto invernadero por dólar de producción, que el promedio de los otros sectores económicos, dejando pasivos ambientales. Los costos ambientales y sociales pueden ser enormes en términos de impactos sobre el suelo, el agua, el aire y los ecosistemas, cuya remediación puede ocasionar mayores costos (Twerefou, 2009).

Por otra parte, la debilidad en la regulación y la falta de compromiso de una gran parte de los empresarios mineros con la responsabilidad socioambiental, sigue ocasionado que las comunidades pierdan confianza en la sostenibilidad de este sector. De acuerdo con el OCMAL (2015), las comunidades no creen en los mineros y en los gobiernos, razón, por la cual, están pendientes de vigilar la calidad del ambiente para evitar posibles desastres, incluso, llegando a oponerse a la ejecución de los proyectos mineros. Al respecto, los actores pueden asumir diversos leguajes de valoración, que van desde una compensación monetaria, equivalente a los daños ambientales sufridos, asumir que el territorio es sagrado, apelar a la defensa de los derechos humanos o a los derechos colectivos indígenas, o insistir en valores ecológicos, que no pueden ser realmente expresados en dinero (Martinez-Alier, 2006).

Los países de la región han afrontado desde diferentes perspectivas estos conflictos, a través del planteamiento de iniciativas, que han permitido mejorar el comportamiento socioambiental de la industria minera; pero, aunque los gobiernos han adoptado políticas para promover el desarrollo de la minería y beneficiarse de la renta que produce, aún se evidencia la ausencia de espacios que permitan acordar con la sociedad civil, su manejo (Cisneros, 2016). Esto es reiterado por la FFNC (2013), para quien la legislación minera va en contravía de los principios de protección del ambiente e incide en el deterioro del medio natural, conllevando a manifestaciones sociales, en contra del desarrollo minero.

Por su parte, los empresarios mineros han realizado esfuerzos para fortalecer la imagen de la industria, a través de la adopción de políticas de responsabilidad social, que buscan mejorar la percepción de las comunidades hacia los proyectos mineros, mostrando su incidencia en el mejoramiento de la calidad de vida y desarrollo de las comunidades. La responsabilidad social empresarial es la herramienta que proporciona a las empresas mineras los mecanismos que permiten integrar y gestionar sus relaciones con los grupos 
de interés clave, buscando compartir los beneficios de desarrollo y proteger los intereses de las empresas (Humphreys, 2000); sin embargo, para la CEPAL (2015a), de acuerdo con las comunidades afectadas, en algunos casos, las acciones desarrolladas por las empresas mineras no han sido del todo satisfactorias.

A pesar de los esfuerzos de los gobiernos y de los empresarios mineros para fortalecer las políticas y las acciones para mejorar el comportamiento social y ambiental de la industria minera, en su mayor parte, se han orientado hacia el manejo ambiental. En este contexto, Everingham (2012) sostiene que, en relación con los impactos que genera la minería, la atención se ha enfocado a la reducción y la mitigación de los daños ambientales y se conoce menos sobre el manejo de los impactos sociales, para asegurar la sostenibilidad de la industria.

La manifestación de los Gobiernos Nacionales de emprender acciones orientadas a superar los conflictos socioambientales que afronta el sector minero, se puede determinar desde los objetivos estratégicos de sus Planes Nacionales de Desarrollo -PND-, evaluando las medidas u orientaciones que se proponen, para promover el sector minero, en particular, en lo referente a las acciones que se plantean para mejorar la tensión que se presenta con las comunidades por los efectos sociales y ambientales que genera la industria. En consecuencia, se trazó como objetivo en este escrito, revisar el comportamiento de la minería en los países de ALC, con enfoque hacia los aspectos socioambientales y la incidencia en su desarrollo, enmarcado en la inclusión de los aspectos socioambientales en los planes de desarrollo nacional, el número y distribución de tales aspectos, relacionados con el desarrollo de actividades mineras y algunos efectos económicos, derivados de estos conflictos, en los proyectos mineros.

\section{MATERIALES Y MÉTODOS}

El análisis de la minería con un enfoque socioambiental, se llevó a cabo en 33 países de ALC (Figura 1), lo cual, requirió una revisión del panorama general de la minería en la región, en el periodo más reciente de los PND, que comprende desde el 2009 al 2017. Las variables utilizadas para este análisis fueron: su importancia en la economía de los países, la existencia de reservas mineras, su inclusión en los PDN, así como las dificultades que afronta el sector minero, a causa de los conflictos ambientales y sociales, que afectan el desarrollo de las actividades mineras. Para ello, se llevaron a cabo cuatro etapas consecutivas a saber.

Se revisó información específica de la minería en cada uno de los países de ALC, en especial, sobre la participación de esta actividad en el PIB y en las exportaciones, con el pro- pósito de identificar la incidencia de la industria minera en la economía, lo que permitió realizar la categorización de los países en términos de la importancia minera en la región. Posteriormente, se desarrolló una revisión de los instrumentos de PND, de cada uno de los países de ALC, con el propósito de identificar cómo han considerado e integrado los aspectos socioambientales relacionados con la minería, estableciendo 4 niveles de inclusión. Para ello, se recurre, principalmente, a información del Observatorio Regional de Planificación para el Desarrollo de ALC, de la CEPAL.

Mediante la utilización de algunos indicadores que permiten determinar el grado de conflictividad social y ambiental de la minería, se evaluó y se comparó la eficacia de las políticas, las estrategias y las acciones, que los países de la región vienen desarrollando, para aminorar dichos conflictos. En esta revisión, se utilizó la información del Observatorio de Conflictos Mineros de América Latina -OCMAL- y estudios e investigaciones realizados a nivel de ALC y en el ámbito Internacional. OCMAL entiende el conflicto minero como las expresiones de descontento, rechazo y resistencia de gente de la academia y de miembros del propio Estado, que surgen como consecuencia del impacto ambiental, económico, social y territorial, que ocasiona el desarrollo de los proyectos mineros. Finalmente, a partir de la revisión de algunos estudios enfocados a la evaluación de los efectos económicos generados por conflictos socioambientales en el desarrollo de proyectos mineros en ALC, se presenta un análisis de las implicaciones de estos conflictos en los costos monetarios de los proyectos y los recursos mineros que se han dejado de extraer por la suspensión temporal y definitiva de proyectos mineros, en algunos países de ALC.

\section{RESULTADOS Y DISCUSIÓN}

Para los estudiosos de temas emergentes, como lo es el enfoque socioambiental aplicado, es claro que la literatura científica sobre la minería, analizada específicamente en las relaciones conjuntas ambiente-sociedad, apenas está iniciando; sin embargo, gracias al esfuerzo de quienes han puesto al servicio del público los observatorios es posible completar revisiones comparativas entre países, como las que aquí se presentan.

En cuanto a la incidencia de la minería en la economía de los países de la región, de acuerdo con la CEPAL (2013a), ALC es la fuente principal de metales y la segunda en petróleo, a nivel mundial: en 2016, participó con el 28\% de la inversión global en exploración minera y el $92 \%$ de esta inversión, se focalizó en 6 países: Chile, Perú, México, Brasil, Argentina y Colombia (S\&P Global, 2017). Específicamente, la minería en la región contribuye, aproximadamente, con el $26 \%$ de la inversión extranjera directa total (CEPAL, 2015b) y participa en un 3,3\% en el producto interno bruto (CEPAL, 2018). A 


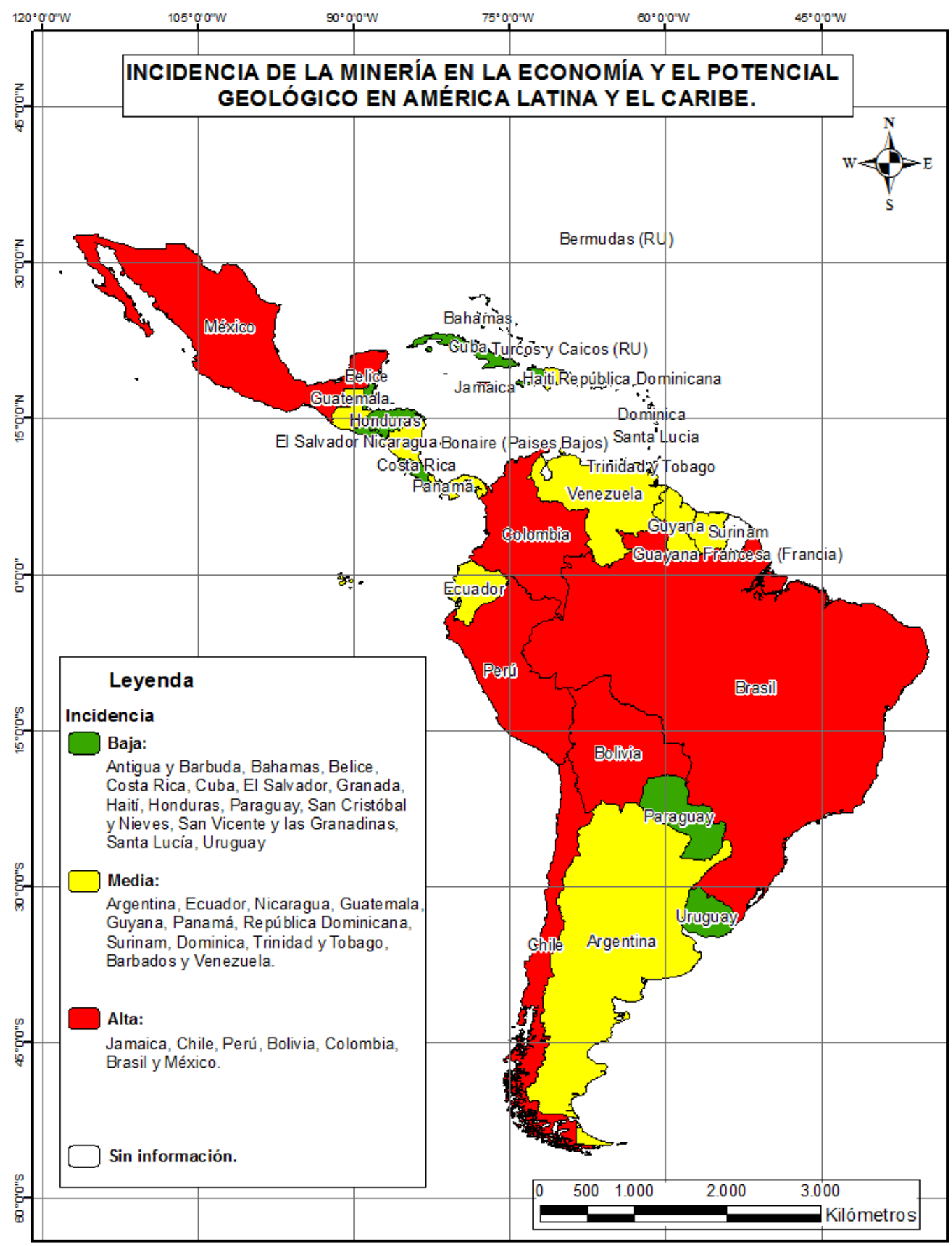

Figura 1. Mapa de categorización de países de ALC, con base en la incidencia de la minería en la economía y el potencial geológico. 
partir del análisis de estos datos, en la tabla 1, se sintetiza la participación en el 2016, de las industrias extractivas, incluyendo petróleo, en el PIB de los países de ALC, así como la participación solo de minerales, en las exportaciones totales.
Con relación a la existencia de reservas mineras, en ALC se destaca que: Brasil tiene cerca del $95 \%$ de las reservas mundiales de niobio; en Chile y Bolivia, se encuentra el $65 \%$ de las reservas de litio; en Perú, Chile y México está el $44 \%$ de las reservas mundiales de cobre y, además, se encuentran reser-

Tabla 1. Participación de la minería en el PIB y exportaciones en países de América Latina y el Caribe.

\begin{tabular}{|c|c|c|c|c|c|c|c|}
\hline \multicolumn{2}{|r|}{ País } & \multirow{2}{*}{$\begin{array}{c}\text { Participación } \\
\text { PIB minero* } \\
2016 \\
0,9 \%\end{array}$} & \multirow{2}{*}{$\begin{array}{c}\begin{array}{c}\text { Participación } \\
\text { exportaciones** } \\
\%\end{array} \\
\text { S.I. }\end{array}$} & \multicolumn{2}{|c|}{ País } & \multirow{2}{*}{$\begin{array}{c}\text { Participación } \\
\text { PIB minero* } \\
2016 \\
0,2 \%\end{array}$} & \multirow{2}{*}{$\begin{array}{c}\begin{array}{c}\text { Participación } \\
\text { exportaciones } * * \\
\%\end{array} \\
\text { S.I. }\end{array}$} \\
\hline 1 & $\begin{array}{l}\text { Antigua y } \\
\text { Bermuda }\end{array}$ & & & 18 & Haití & & \\
\hline 2 & Argentina & $4,0 \%$ & $<1 \%$ & 19 & Honduras & $0,6 \%$ & $<1 \%$ \\
\hline 3 & Bahamas & $0,9 \%$ & S.I. & 20 & Jamaica & $1,7 \%$ & $45,9 \%$ \\
\hline 4 & Barbados & $0,2 \%$ & $3,6 \%$ & 21 & México & $3,9 \%$ & $<1 \%$ \\
\hline 5 & Belice & $0,5 \%$ & $<1 \%$ & 22 & Nicaragua & $3,0 \%$ & $<1 \%$ \\
\hline 6 & Bolivia & $10,4 \%$ & $34,7 \%$ & 23 & Panamá & $2,2 \%$ & $<1 \%$ \\
\hline 7 & Brasil & $1,1 \%$ & $7,3 \%$ & 24 & Paraguay & $0,2 \%$ & $<1 \%$ \\
\hline 8 & Chile & $8,9 \%$ & $44,0 \%$ & 25 & Perú & $9,0 \%$ & $42,1 \%$ \\
\hline 9 & Colombia & $5,9 \%$ & $14,9 \%$ & 26 & $\begin{array}{l}\text { República } \\
\text { Dominicana }\end{array}$ & $2,0 \%$ & S.I. \\
\hline 10 & Costa Rica & $0,3 \%$ & $<1 \%$ & 27 & $\begin{array}{l}\text { Saint Cristobal } \\
\text { y Nevis }\end{array}$ & $0,1 \%$ & S.I. \\
\hline 11 & Cuba & $0,6 \%$ & $2,5 \%$ & 28 & $\begin{array}{l}\text { San Vicente } \\
\text { y las Grana- } \\
\text { dinas }\end{array}$ & $0,2 \%$ & S.I. \\
\hline 12 & Dominica & $1,5 \%$ & $9,4 \%$ & 29 & Santa Lucía & $0,4 \%$ & S.I. \\
\hline 13 & Ecuador & $4,6 \%$ & $<1 \%$ & 30 & Suriname & $6,6 \%$ & S.I. \\
\hline 14 & El Salvador & $0,3 \%$ & $<1 \%$ & 31 & $\begin{array}{l}\text { Trinidad y } \\
\text { Tobago }\end{array}$ & $9,2 \%$ & $2,5 \%$ \\
\hline 15 & Granada & $0,2 \%$ & S.I. & 32 & Uruguay & $0,5 \%$ & $<1 \%$ \\
\hline 16 & Guatemala & $1,5 \%$ & $5,3 \%$ & 33 & Venezuela & $28,4 \%$ & $0,3 \%$ \\
\hline 17 & Guyana & $16,8 \%$ & S.I. & & & & \\
\hline
\end{tabular}

*Incluye Petróleo. ** Solo minería.

vas importantes de molibdeno, estaño, zinc, aluminio, boro, hierro, antimonio, bismuto, carbón, níquel, manganeso, cromo, plomo, que mueven la economía global (BID, 2016).

En la figura 1, se observa el resultado de analizar la información sobre participación de la minería en el PIB y en las exportaciones, así como la existencia de reservas mineras, con lo cual, se logró establecer la siguiente categorización de los países de ALC, en términos de la importancia en la economía y potencial minero.
Importancia Alta. Países, en los que se cumple, al menos, dos, de los tres siguientes requisitos: i) participación de la minería en el PIB $>3,5$, ii) participación en las exportaciones $>5 \%$ y iii) con reservas importantes en algún mineral. Corresponde a 7 países: Jamaica, Chile, Perú, Bolivia, Colombia, Brasil y México.

Importancia Media. Países, en los que la participación de la minería en el PIB sea mayor que $1 \%$ y menor que 3,5\%. 12 países: Argentina, Ecuador, Nicaragua, Guatemala, Guyana, Panamá, República Dominicana, Suriname, Dominica, Trinidad y Tobago, Barbados y Venezuela. 
Importancia Baja. Países, en los que la participación de la minería en el PIB es menor al 1\%. 14 países: Antigua y Barbuda, Bahamas, Belice, Costa Rica, Cuba, El Salvador, Granada, Haití, Honduras, Paraguay, San Cristóbal y Nevis, San Vicente y las Granadinas, Santa Lucía y Uruguay.

Como se observa en la figura 2 y el cuadro 1, en el análisis del nivel de inclusión de los aspectos socioambientales relacionados con la minería, al interior de los PND de los países de ALC, se pudieron establecer cuatro niveles: nulo, bajo, medio y alto.
Nivel Nulo. En su plan nacional de desarrollo no se consideran las actividades mineras. Son 6 países: Antigua y Barbudas, Belice, Costa Rica, San Vicente y las Granadinas, Santa Lucía y Trinidad y Tobago.

Nivel Bajo. En su plan nacional de desarrollo, se mencionan las actividades mineras, pero no se establecen objetivos estratégicos específicos relacionados con los aspectos socioambientales de la minería. Son tres países: Cuba, Honduras y Surinam.

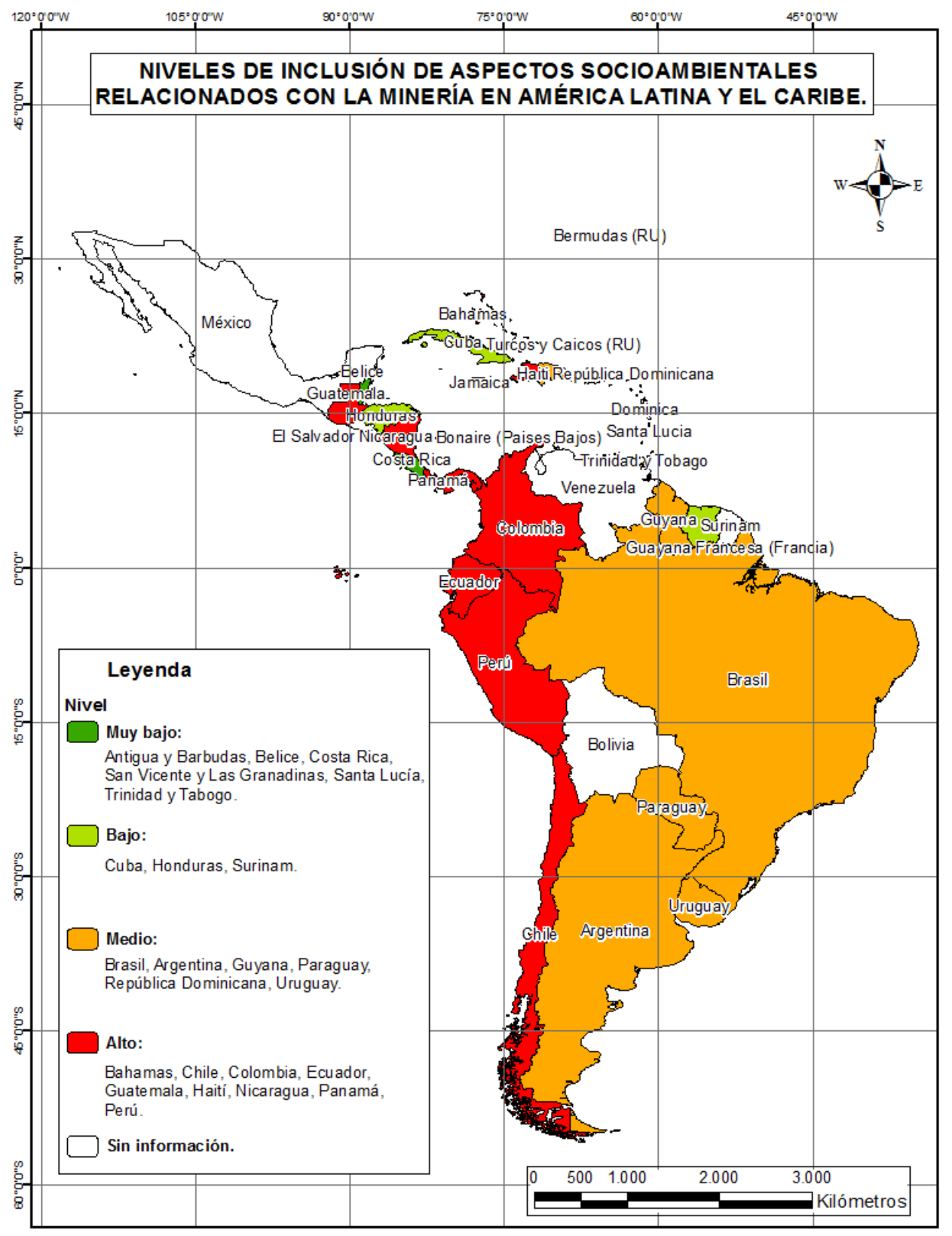

Figura 2. Niveles de inclusión de aspectos socioambientales relacionados con la minería en América Latina y el Caribe. 
Cuadro 1. Aspectos socioambientales relacionados con la minería en los PDN de los países de ALC.

\begin{tabular}{|c|c|c|}
\hline País & Autor & Aspectos socioambientales \\
\hline $\begin{array}{l}\text { Antigua y } \\
\text { Barbudas }\end{array}$ & $\begin{array}{l}\text { Ministries of Government of } \\
\text { Antigua and Barbuda and Statutory } \\
\text { Organizations of the Public Sector } \\
\text { (2016) }\end{array}$ & No plantea objetivos para minería. \\
\hline Argentina & Mauricio Macri (2015) & $\begin{array}{l}\text { La minería argentina tiene un gran potencial. Para desarrollarlo, el camino es el } \\
\text { Acuerdo Federal Minero en marcha, sobre temas productivos, tributarios, ambien- } \\
\text { tales, comunitarios y normativos. }\end{array}$ \\
\hline Bahamas & $\begin{array}{l}\text { Comité Directivo del Plan Nacional de } \\
\text { Desarrollo (2016) }\end{array}$ & $\begin{array}{l}\text { Crear condiciones para atraer grandes flujos de inversión minera, con el requisito } \\
\text { que las inversiones produzcan sostenibilidad social y progreso económico para } \\
\text { todos las personas y para el ambiente. }\end{array}$ \\
\hline Barbados & & No hay datos al respecto. \\
\hline Belice & $\begin{array}{l}\text { Government of Belize, Ministry of } \\
\text { Economy Development (2016) }\end{array}$ & No plantea objetivos para minería. \\
\hline Bolivia & $\begin{array}{l}\text { Estado Plurinacional de Bolivia. } \\
\text { Ministerio de Planificación del } \\
\text { Desarrollo (2016) }\end{array}$ & $\begin{array}{l}\text { Bolivia habrá consolidado un sector hidrocarburífero y minero con un significativo } \\
\text { incremento de las reservas naturales de gas y de los recursos mineros y metales, } \\
\text { garantizando que, en su producción, se utilicen las mejores tecnologías disponibles, } \\
\text { para prevenir, mitigar y remediar los daños causados y para restaurar los compo- } \\
\text { nentes y las zonas de vida de la Madre Tierra, que resultan de estas actividades. }\end{array}$ \\
\hline Brasil & $\begin{array}{l}\text { Governo Federal Brasil. Ministério } \\
\text { do Planejamento, Orçamento e } \\
\text { Gestão Secretaria de planejamento e } \\
\text { investimento estratégico (2016) }\end{array}$ & $\begin{array}{l}\text { Ampliación del conocimiento geológico, la gestión de los minerales estratégicos, } \\
\text { la agregación de valor con competitividad, la gobernanza pública eficaz y la pro- } \\
\text { ducción sostenible. }\end{array}$ \\
\hline Chille & Sebastian Piñera Echenique (2017) & $\begin{array}{l}\text { Desarrollar una minería más sustentable ambientalmente e inclusiva socialmente. } \\
\text { Crear mecanismos de diálogo y solución temprana de conflictos entre empresas y } \\
\text { comunidades, que mejore la confianza y dinamice los proyectos mineros y energé- } \\
\text { ticos. Incentivar los aportes de las empresas mineras a municipalidades, ONG's y } \\
\text { comunidades vecinas. }\end{array}$ \\
\hline Colombia & $\begin{array}{l}\text { República de Colombia. Departamento } \\
\text { Nacional de Planeación (2015) }\end{array}$ & $\begin{array}{l}\text { Consolidar al sector minero como impulsor del desarrollo sostenible del país, con } \\
\text { responsabilidad social y ambiental. }\end{array}$ \\
\hline Costa Rica & $\begin{array}{l}\text { Gobierno de Costa Rica. Ministerio } \\
\text { de Planificación Nacional y Política } \\
\text { Económica (2014) }\end{array}$ & No plantea objetivos para minería. \\
\hline Cuba & Congreso Cuba (2016) & $\begin{array}{l}\text { Plantea un eje estratégico de Recursos Naturales y Medio Ambiente, que no esta- } \\
\text { blece ningún objetivo relacionado con el desarrollo de las actividades mineras. }\end{array}$ \\
\hline Dominica & Commonwealth of Dominica (2014) & $\begin{array}{l}\text { Implementar el Código de Prácticas para Canteras, para el uso sostenible de la } \\
\text { tierra con fines extractivos, así como la gestión ambiental a largo plazo. }\end{array}$ \\
\hline Ecuador & $\begin{array}{l}\text { Secretaría Nacional de Planificación y } \\
\text { Desarrollo (2017) }\end{array}$ & $\begin{array}{l}\text { Realizar el ordenamiento de las actividades de exploración y explotación de recur- } \\
\text { sos naturales no renovables que se desarrollen, a nivel nacional, con énfasis en la } \\
\text { Amazonía y la zona costera, la plataforma continental, suelo y subsuelo marina, } \\
\text { para minimizar externalidades sociales y ambientales. }\end{array}$ \\
\hline El Salvador & $\begin{array}{l}\text { Secretaría Técnica y de Planificación } \\
\text { Gobierno de El Salvador (2015) }\end{array}$ & $\begin{array}{l}\text { Fortalecer el marco normativo e institucional para regular actividades de alto } \\
\text { riesgo, como la minería metálica, para reducir los niveles de contaminación e } \\
\text { insalubridad ambiental, para contribuir a mejorar la salud de la población y el fun- } \\
\text { cionamiento efectivo de los ecosistemas. }\end{array}$ \\
\hline Granada & $\begin{array}{l}\text { Agency for Reconstruction and } \\
\text { Development Inc (2007) }\end{array}$ & $\begin{array}{l}\text { Promover la conciencia y el compromiso con las consideraciones ambientales, en } \\
\text { extracciones de arena. }\end{array}$ \\
\hline Guatemala & $\begin{array}{l}\text { Consejo Nacional de Desarrollo Urbano } \\
\text { y Rural 2012-2016 (2014) }\end{array}$ & $\begin{array}{l}\text { Lograr que el aprovechamiento de recursos naturales no renovables (minería y } \\
\text { petróleo) se haga únicamente bajo los lineamientos de un ordenamiento territorial, } \\
\text { que asegure la sostenibilidad ambiental, respete los derechos de los Mayas, Xinkas } \\
\text { y Garífunas, mejore las condiciones de vida de las poblaciones locales y garantice } \\
\text { condiciones dignas para la clase trabajadora. }\end{array}$ \\
\hline
\end{tabular}


Continuación Cuadro 1

\begin{tabular}{|c|c|c|}
\hline Guyana & Ministry of the Presidency (2017) & $\begin{array}{l}\text { Fortalecimiento y modernización de los marcos legales e institucionales para } \\
\text { sectores clave, como minería, entre otros, para crear un entorno propicio para la } \\
\text { inversión en infraestructura y prácticas resilientes y sostenibles. }\end{array}$ \\
\hline Haití & $\begin{array}{l}\text { Gouvernement de la République } \\
\text { D'HAïTI. Ministère de la Planification et } \\
\text { de la Coopération externe (2012) }\end{array}$ & $\begin{array}{l}\text { Promover aprovechamiento de los recursos minerales para maximizar los } \\
\text { beneficios de este negocio en la economía del país, para garantizar su explotación } \\
\text { racional, garantizar la protección de la salud y la seguridad de los trabajadores y la } \\
\text { población, y protección del medioambiente. }\end{array}$ \\
\hline Jamaica & Planning Institute of Jamaica (2009) & $\begin{array}{l}\text { Proporcionar un enfoque integrado para la planificación del uso de la tierra, la } \\
\text { gestión de las reservas y el desarrollo de la infraestructura para el sector; ayudar a } \\
\text { construir comunidades mineras viables; mejorar la rehabilitación de tierras mina- } \\
\text { das; y promover un mayor cumplimiento de las normas y legislación ambientales. }\end{array}$ \\
\hline México & Gobierno de la República (2013) & $\begin{array}{l}\text { Mantener el dinamismo y la competitividad del sector minero en un ambiente de } \\
\text { volatilidad en los precios internacionales; beneficiar y respetar los derechos de las } \\
\text { comunidades o municipios donde se encuentran las minas, así como aumentar } \\
\text { los niveles de seguridad. }\end{array}$ \\
\hline Nicaragua & $\begin{array}{l}\text { Gobierno de Reconciliación y Unidad } \\
\text { Nacional (2012) }\end{array}$ & $\begin{array}{l}\text { Ampliación de la minería industrial y artesanal de forma sostenible ambiental y } \\
\text { socialmente, bajo la responsabilidad del sector público y de la inversión privada } \\
\text { directa. }\end{array}$ \\
\hline Panamá & $\begin{array}{l}\text { Gobierno de la República de Panamá } \\
\text { (2014) }\end{array}$ & $\begin{array}{l}\text { Promover la minería responsable, con provecho equitativo para todos los actores: } \\
\text { la comunidad local, el país en general y quienes han invertido capital de riesgo. }\end{array}$ \\
\hline Paraguay & $\begin{array}{l}\text { Gobierno Nacional. Secretaría Técnica } \\
\text { de Planificación del Desarrollo } \\
\text { Económico y Social (2014) }\end{array}$ & $\begin{array}{l}\text { Fortalecer la capacidad de prospección y exploración de los recursos mineros e } \\
\text { hidrocarburos, y asegurar la explotación sostenible. }\end{array}$ \\
\hline Perú & $\begin{array}{l}\text { CEPLAN - Centro Nacional de } \\
\text { Planeamiento Estratégico (2011) }\end{array}$ & $\begin{array}{l}\text { Estimular la exploración y explotación minera, con el enfoque de responsabilidad } \\
\text { social, ambiental y sustentable, que preconiza la integración densa de la economía } \\
\text { regional con las inversiones primario extractivas, a través de cadenas productivas } \\
\text { y/o portafolios de inversiones diversificadas de las grandes empresas. }\end{array}$ \\
\hline Santa Lucia & $\begin{array}{l}\text { International Design and Entertainment } \\
\text { Associates Incorporated (2008) }\end{array}$ & Los objetivos del plan no contemplan dentro de su planeación la minería. \\
\hline Suriname & $\begin{array}{l}\text { Government of the Republic of } \\
\text { Suriname (2017) }\end{array}$ & $\begin{array}{l}\text { Optimizar la riqueza mineral para apoyar crecimiento económico estable y } \\
\text { equilibrado. No especifica objetivos socioambientales. }\end{array}$ \\
\hline $\begin{array}{l}\text { Trinidad y } \\
\text { Tobago }\end{array}$ & $\begin{array}{l}\text { Government of the Republic of Trinidad } \\
\text { and Tobago (2016) }\end{array}$ & No contempla dentro de su planeación la minería. \\
\hline Uruguay & $\begin{array}{l}\text { Presidencia, Oficina de Planeamiento y } \\
\text { Presupuesto (2017) }\end{array}$ & $\begin{array}{l}\text { Se pondrá una mirada de futuro, tanto sobre los aspectos productivos, tecnológi- } \\
\text { cos, ambientales y geopolíticos como sobre los asuntos ligados al mercado de } \\
\text { trabajo, al desarrollo de los territorios y a las particularidades socioculturales. }\end{array}$ \\
\hline Venezuela & Nicolás Maduro Moros (2013) & $\begin{array}{l}\text { Incrementar la extracción de minerales estratégicos en los yacimientos identifica- } \\
\text { dos y potenciales, favoreciendo el uso de tecnologías que maximicen su aprove- } \\
\text { chamiento y preserve la vida humana. }\end{array}$ \\
\hline
\end{tabular}


Nivel medio. En su plan nacional de desarrollo, se mencionan las actividades mineras, pero se establecen objetivos estratégicos generales de producción minera sostenible. Corresponde a 6 países: Brasil, Argentina, Guyana, Paraguay, República Dominicana y Uruguay o establecen solo para uno de los componentes, ambiental, 5 países: Bolivia, Dominica, El Salvador, Granada y Jamaica; o social, 2 países: México y Venezuela.

Nivel Alto: En su plan nacional de desarrollo, se establecen objetivos estratégicos para promover el sector minero, especificando claramente la necesidad de desarrollar una minería, que integre los aspectos ambientales y sociales, 9 países: Bahamas, Chile, Colombia, Ecuador, Guatemala, Haití, Nicaragua, Panamá y Perú.

La revisión de los PND permite concluir que, únicamente el $29 \%$ de los países de ALC, consideran, dentro de sus objetivos estratégicos, la inclusión de los aspectos socioambientales, relacionados con la minería; el $42 \%$, los incluyen de manera parcial; el $1 \%$, de manera incipiente y el $20 \%$, no los tienen en cuenta. Por lo tanto, al comparar el indicador de categoría de importancia de la minería en los países de ALC, con los niveles de inclusión de los aspectos socioambientales en los PND, se observa que, de los países categorizados de relevancia minera alta, es decir, Bolivia, Brasil, Chile, Colombia, Jamaica, México y Perú, únicamente Chile, Colombia y Perú, que representan el $10 \%$ de los países de ALC, tienen identificada la prioridad de promover la minería, con criterios de responsabilidad socioambiental; no obstante, países, como Bahamas y Haití, por la poca relevancia de las actividades mineras en su economía y su bajo potencial geológico, identifican en sus PDN, objetivos concretos para el desarrollo de estas actividades, de manera responsable, en el aspecto socioambiental.

Por otra parte, el alto potencial que tiene la minería para transformar condiciones del ambiente, las comunidades y la economía, se manifiesta en conflictos o disputas entre las comunidades y los inversionistas mineros (Davis \& Franks, 2011). Por una parte, la minería genera beneficios socioeconómicos y, por otra, los impactos ambientales y conflictos sociales (University of Eastern Finland, 2012). En la revisión correspondiente a los conflictos relacionados con el desarrollo de actividades mineras en ALC, de acuerdo con OCMAL (2018), en esta región, se ha publicado información sobre 254 conflictos, que involucran a 264 proyectos mineros, de los cuales, seis corresponden a conflictos transfronterizos (Figura 3); en el 60\% de los países de ALC, se ha reportado la publicación de conflictos socioambientales relacionados con la minería. En solo 6 países, se acumula el $81 \%$ de los conflictos totales: México, 17\%; Chile, 16\%; Perú, 15\%; Argentina, $11 \%$; Brasil $10 \%$ y Colombia, $6 \%$ que, en términos generales, corresponden a los países de mayor importancia minera en ALC.
Al relacionar el porcentaje de conflictos con la incorporación de políticas específicas sobre aspectos socioambientales mineros en los PDN, se observa, que Chile, Colombia y Perú han interiorizado la necesidad de mejorar el comportamiento socioambiental de la actividad minera, mientras que en Brasil, en México y en Argentina, las políticas gubernamentales aún no son claras sobre la necesidad de fortalecer los estándares socioambientales de la minería, como un mecanismo para legitimar el desarrollo de esta actividad en los aspectos socioambientales, que disminuya la conflictividad con las comunidades y los territorios.

Las reacciones de las comunidades a los impactos socioambientales que generan los proyectos mineros, se convierten en costos significativos para las empresas, las comunidades y los gobiernos (Davis \& Franks, 2014). En relación con la magnitud de los costos financieros que han ocasionado los conflictos socioambientales en ALC, Franks et al. (2014) reportan que, en 2010, un retraso de 9 meses en la construcción en una mina ubicada en Latinoamérica, generó costos adicionales por US\$750 millones, que corresponden a cerca de US\$20 millones por semana. Puntualmente, en Argentina, en 2006, como consecuencia de conflictos socioambientales, los desarrolladores de un proyecto minero perdieron US\$379 millones en activos y se desperdiciaron US\$1.330 millones, correspondientes a las reservas proyectadas del mineral que se iba a explotar (Herz et al. 2007). En 2003, en Perú, por la imposibilidad de desarrollar un proyecto, se reportó la pérdida de inversión de US\$9,3 millones y, adicionalmente, las reservas valoradas en ese momento, en US\$ 53 millones (Manhattan Minerals Corp, 2004). En 2014, en Perú, Pinedo (2014) reporta que, como consecuencia de un conflicto socioambiental, aumentó el costo estimado de un proyecto, de US\$3.3 mil millones a US\$5 mil millones.

El costo de los conflictos socioambientales genera pérdidas empresariales, riesgo en el desarrollo de proyectos mineros, límites a la inversión extranjera, disminución de los ingresos de los Estados, que deben ser redistribuidos en la atención de necesidades básicas. Los gobiernos deben liderar la generación de políticas públicas, que se vean reflejadas en sus PND, orientadas a reducir los conflictos socioambientales relacionados con la minería, mediante el trabajo concertado con los empresarios y comunidades, que garanticen la información adecuada, a estas últimas, sobre las implicaciones económicas, sociales, ambientales y culturales de los proyectos. Deben promover un relacionamiento desde las etapas tempranas, que permita concertar el desarrollo de las actividades mineras con los entes municipales locales, que asegure beneficios económicos para todas las partes. Viscidi \& Fargo (2015) señalan que en países, como Perú, Colombia, México y Chile, los gobiernos no tienen procesos claros ni capacidad política para manejar los conflictos socioambientales y plantean una serie de sugerencias para enfrentar 


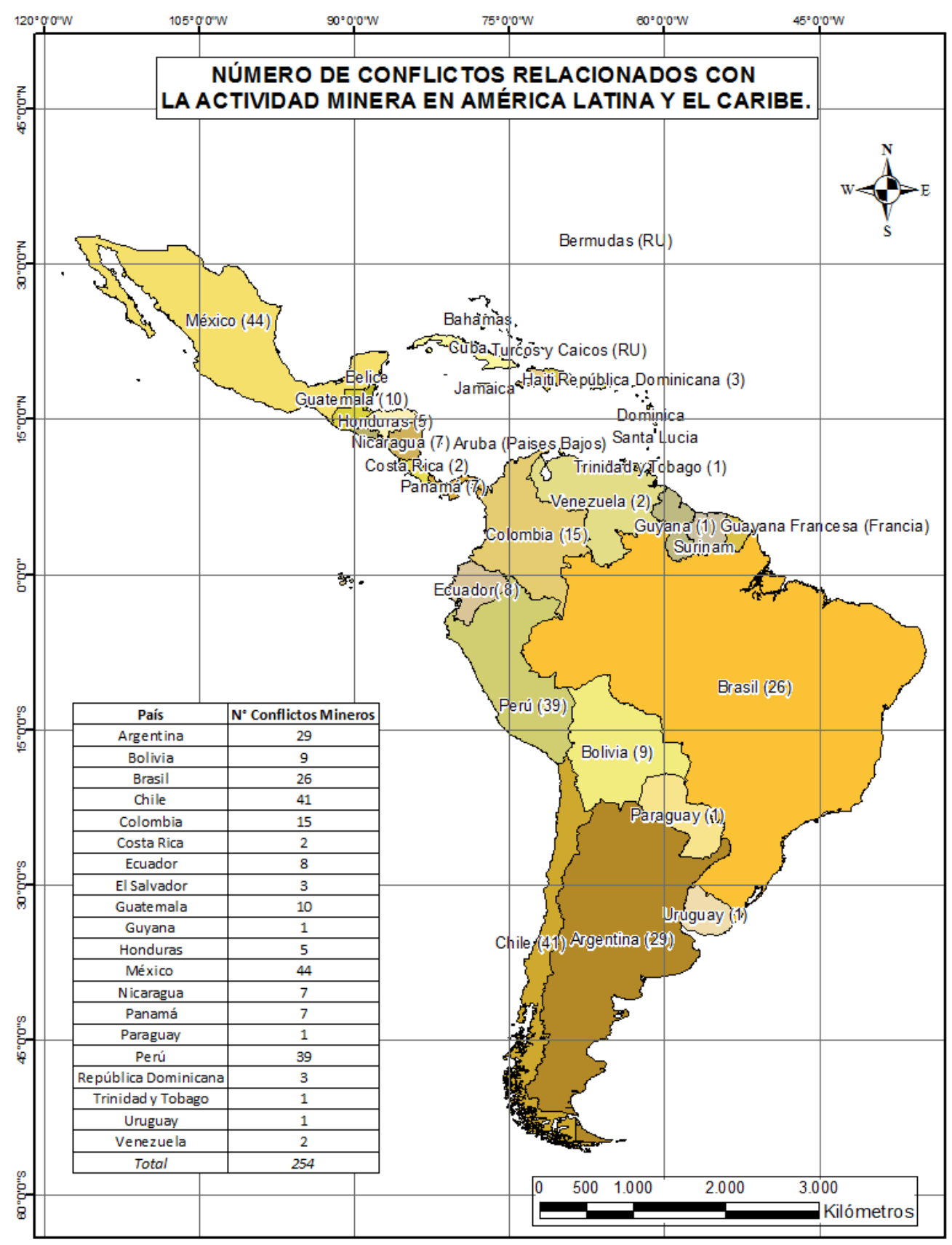

Figura 3. Conflictos relacionados con la actividad minera en América Latina y el Caribe.

la situación en diferentes instancias, destacando la necesidad de equilibrar el interés nacional por el aprovechamiento de los recursos naturales con el interés local; equilibrar la regulación de la industria, que permita la inversión y asegure la protección ambiental y cultural y, finalmente, promover que la inversión y los ingresos fiscales, a largo plazo, incidan en el desarrollo económico local.
Por otro lado, es importante que las empresas mineras internalicen en sus análisis costo beneficio, los riesgos socioambientales, como generadores de conflictos, que pueden causar la suspensión parcial o definitiva de los proyectos mineros, que se traducen en sobrecostos para los proyectos e incertidumbre a los países, en los que la renta minera soporta su economía. La responsabilidad social empresarial 
podría ser el vehículo que permita a las empresas mineras pacificar a las comunidades locales y minimizar los conflictos socioambientales, mediante el cambio en el pensamiento de responsabilidad corporativa, por el de responsabilidad recíproca (Abuya, 2016). Según el BID (2016), la dinámica de los conflictos socioambientales relacionados con la minería es tan compleja, que la prevención de los mismos requiere de políticas integrales que fortalezcan el marco regulatorio y la capacidad institucional, soportado en el esfuerzo concertado de las partes interesadas, bajo el principio del respeto y la confianza mutua. Estas políticas, necesariamente, se deben involucra, de manera efectiva, en los instrumentos de planeación nacional, con el propósito de asegurar la intervención acertada de los gobiernos, para promover el crecimiento sostenible de la minería, en un marco que se fundamente en el respeto por el ambiente, por las comunidades y genere beneficios a los inversionistas, la población y los países. En esta vía, Viana et al. (2005) señalan que la minería desarrollada con bajos estándares, genera impactos socioambientales, a pesar de los posibles beneficios que puede traer, frente a lo que sugieren conciliar los conflictos por apropiación y uso del suelo, para un mejor aprovechamiento sostenible de los recursos descubiertos y en proceso de explotación.

Es evidente que el desarrollo de las actividades mineras incide en la economía local, en los usos del suelo, afecta al ambiente y a la sociedad en general, razón, por la cual, el ordenamiento territorial es el instrumento que posibilita, a través de la concertación entre gobierno, empresas y sociedad, armonizar los intereses entre las partes, mediante procesos transparentes y de respeto mutuo, que legitime el desarrollo de la minería, buscando beneficios socioambientales y económicos, de nivel local y nacional. Una posible salida, se encuentra en el ordenamiento territorial, ya que propicia conciliar y racionalizar los intereses entre el aprovechamiento de los recursos naturales por parte de los inversionistas mineros y la necesidad de conservar el ambiente y mejorar las condiciones socioeconómicas de los territorios (Viana, 1997).

A pesar que la minería es indispensable para el desarrollo de los países de ALC, la mayor parte de los gobiernos de la región aún no han interiorizado la necesidad de abordar, adecuadamente, el reto de promover políticas socioambientales integrales, que se vean reflejadas en sus PND y sean precisas y contundentes frente a la necesidad de promover el desarrollo de una minería, soportada en el diálogo entre el gobierno, las empresas y las comunidades y en la aplicación de buenas prácticas ambientales y sociales, como la responsabilidad social empresarial, que consolide la legitimidad del sector en todos los niveles territoriales.

Conflictos de intereses: El manuscrito fue preparado y revisado por el autor, quien declara que no existe conflicto de intereses que ponga en riesgo la validez de los resultados presentados.

\section{REFERENCIAS}

1. ABUYA, W.O. 2016. Mining conflicts and Corporate Social Responsibility: Titanium mining in Kwale, Kenya. The Extractive Industries and Society. (Netherlands). 9p. Available at: https://kundoc.com/ pdf-mining-conflicts-and-corporate-social-responsibility-titanium-mining-in-kwale-ke.html (con acceso 19/05/18).

2. AGENCY FOR RECONSTRUCTION AND DEVELOPMENT INC. 2007. National Strategic Development Plan Grenada. 155p. Available at: http://www.ilo. org/dyn/youthpol/es/equest.fileutils.docHandle?p_ uploaded_file_id=577 (con acceso 10/05/17).

3. BANCO INTERAMERICANO DE DESARROLLO -BID-. 2016. Extractives in Latin America and the Caribbean. The Basics. Technical Note No. IDB-TN-907. (EEUU). 20p.

4. BANCO MUNDIAL. 2012. Wealth and Sustainability: The Environmental and Social Dimensions of the Mining Sector in Peru. Main Report (2). 194p.

5. CENTRO NACIONAL DE PLANEAMIENTO ESTRATÉGICO -CEPLAN-. 2011. Plan Bicentenario. El Perú hacia el Siglo XXI. (Perú). 265p. Disponible desde Internet en: https:/observatorioplanificacion.cepal. org/sites/default/files/plan/files/plan_bicentenario_ ceplan_index.pdf (con acceso 16/05/18).

6. CISNEROS, P. 2016. Política minera y sociedad civil en América Latina. Editorial IAEN, 1 edición. (Ecuador). 404p.

7. COMISIÓN ECONÓMICA PARA AMÉRICA LATINA Y EL CARIBE -CEPAL-. 2010. El desarrollo sostenible en América Latina y El Caribe: tendencias, avances y desafíos en materia de consumo y producción sostenibles, minería, transporte, productos químicos y gestión de residuos. (Chile). 133p.

8. COMISIÓN ECONÓMICA PARA AMÉRICA LATINA Y EL CARIBE -CEPAL-. 2013a. Recursos Naturales en UNASUR, situación y tendencias para una Agenda de Desarrollo Regional. (Chile). 108p.

9. COMISIÓN ECONÓMICA PARA AMÉRICA LATINA Y EL CARIBE -CEPAL-. 2013b. Natural resources: status 
and trends towards a regional development agenda in Latin America and the Caribbean. (Chile). 104p.

10. COMISIÓN ECONÓMICA PARA AMÉRICA LATINA Y EL CARIBE -CEPAL-. 2015a. Desarrollo minero y conflictos socioambientales. Los casos de Colombia, México y el Perú. (Chile). 57p.

11. COMISIÓN ECONÓMICA PARA AMÉRICA LATINA Y EL CARIBE -CEPAL-. 2015b. La Inversión Extranjera Directa en América Latina y el Caribe. 144p.

12. COMISIÓN ECONÓMICA PARA AMÉRICA LATINA Y EL CARIBE -CEPAL-. 2018. CEPALSTAT. Bases de Datos y Publicaciones Estadísticas Comisión Económica para América Latina y el Caribe. Disponible desde Internet en: http://estadisticas.cepal.org/cepalstat/ perfilesNacionales.html?idioma $=$ spanish (con acceso $28 / 04 / 18)$.

13. COMITÉ DIRECTIVO DEL PLAN NACIONAL DE DESARROLLO. 2016. The National Development Plan of the Bahamas. 460p. Available at: http://vision2040bahamas.org/media/uploads/Draft_National_ Development_Plan_01.12.2016_for_public_release. pdf (con acceso 16/05/18).

14. COMMONWEALTH OF DOMINICA. 2014. Fourth Medium-Term Growth and Social Protection Strategy 2014-2018. Towards Economic Transformation: A Pathway to Sustainable Development. 82p. Available at: https://observatorioplanificacion.cepal.org/sites/ default/files/plan/files/DominicaGrowthandSocialProtectionStrategy.pdf (con acceso 27/05/18).

15. CONGRESO CUBA. 2016. Plan Nacional de Desarrollo Económico y Social 2030 de Cuba: propuesta de visión de la Nación, ejes y sectores estratégicos. 32p. Disponible desde Internet en: http://repositorio. geotech.cu/xmlui/bitstream/handle/1234/669/ Plan\%20nacional\%20de\%20desarrollo\%20 económico\%20y\%20social\%20hasta\%202030. pdf?sequence $=1 \&$ isAllowed $=y \quad($ con $\quad$ acceso 25/05/18).

16. CONSEJO NACIONAL DE DESARROLLO URBANO Y RURAL. 2014. Plan Nacional de Desarrollo: K`atun Nuestra Guatemala 2032. 480p. Disponible desde internet en: https://observatorioplanificacion. cepal.org/sites/default/files/plan/files/ GuatemalaPlanNacionaldeDesarrollo2032.pdf (con acceso 13/05/18).
17. DAVIS, R.; FRANKS, D.M. 2011. The costs of conflict with local communities in the extractive industry. SRMINING 2011. First International Seminar on Social Responsibility in Mining (6). (Chile). 13p. Available at: https://www.csrm.uq.edu.au/Portals/0/11srm cap06_p88.pdf (con acceso 5/05/18).

18. DAVIS, R.; FRANKS, D.M. 2014. Costs of Company-Community Conflict in the Extractive Sector. (EEUU). 56p. Available at: https://sites.hks.harvard.edu/m-rcbg/ CSRI/research/Costs\%20of\%20Conflict_Davis\%20 \%20Franks.pdf (con acceso 13/05/18).

19. ESTADO PLURINACIONAL DE BOLIVIA, MINISTERIO DE PLANIFICACIÓN DEL DESARROLLO. 2016. Plan de Desarrollo Económico y Social. Estado Plurinacional de Bolivia en el marco del Desarrollo Integral para Vivir Bien, 2016-2020. 204p. Disponible desde Internet en: https://observatorioplanificacion. cepal.org/sites/default/files/plan/files/pdes20162020.pdf (con acceso 26/05/18).

20. ESPLUGA, T.J.; LEMKOW, L. 2017. Sociología ambiental Pensamiento socioambiental y ecología social del riesgo. Ed. Iscaria. (España). 280p.

21. EVERINGHAM, J.A. 2012. Toward social of mining. Greener Management International. (Australia). Greenleaf Publishing: p.91-103.

22. FLORIANI, D. 2014. Nuevos sentidos para una ciencia socioambiental desde la perspectiva del pensamiento complejo: algunas reflexiones. (Brasil). Revista Líder 24:9-31.

23. FRANKS, D.M.; DAVIS, R.; BEBBINGTOND, A.J.; ALI, S.H.; KEMP, D.; SCURRAH, M. 2014. Conflict translates environmental and social risk into business costs. Sustainability Science (EEUU). 111(21):75767581. https://doi.org/10.1073/pnas.1405135111

24. FUNDACIÓN FORO NACIONAL POR COLOMBIA FFNC-. 2013. La normativa minera en países de América Latina, un estudio sobre Bolivia, Brasil, Chile, Colombia, Ecuador, México y Perú. Informe Regional. 19p.

25. GOBIERNO DE COSTA RICA. MINISTERIO DE PLANIFICACIÓN NACIONAL Y POLÍTICA ECONÓMICA. 2014. Plan Nacional de Desarrollo 2015-2018. 560p. Disponible desde Internet en: https:/observatorioplanificacion.cepal.org/sites/ default/files/plan/files/Costa\%20Rica\%20Plan\%20 
nacional\%20de\%20Desarrollo\%202015\%20 2018\%20lite.pdf (con acceso 26/05/18).

26. GOBIERNO DE LA REPÚBLICA DE MÉXICO. 2013. Plan Nacional de Desarrollo 2013-2018. 183p. Disponible desde Internet en: https://observatorioplanificacion. cepal.org/sites/default/files/plan/files/ MexicoPlanNacionaldeDesarrollo20132018.pdf (con acceso 25/05/18).

27. GOBIERNO DE LA REPÚBLICA DE PANAMÁ. 2014. Plan Estratégico de Gobierno 2015-2019. 138p. Disponible desde Internet en: https:// observatorioplanificacion.cepal.org/sites/default/ files/plan/files/PanamaPlanEstrategicodeGobierno. pdf (con acceso 12/05/18).

28. GOBIERNO NACIONAL PARAGUAY. SECRETARÍA TÉCNICA DE PLANIFICACIÓN DEL DESARROLLO ECONÓMICO Y SOCIAL. 2014. Plan Nacional de Desarrollo 2030. 87p. Disponible desde Internet en: https://observatorioplanificacion.cepal.org/sites/ default/files/plan/files/ParaguayPlanNacionaldeDesarrollo2030.pdf (con acceso 26/05/18).

29. GOBIERNO DE RECONCILIACIÓN Y UNIDAD NACIONAL NICARAGUA. 2012. Plan Nacional de Desarrollo Humano 2012-2016. 203p. Disponible desde Internet en: https://observatorioplanificacion. cepal.org/sites/default/files/plan/files/ NicaraguaPlanNacionaldeDesarrolloHumano.pdf (con acceso 12/05/18).

30. GOUVERNEMENT DE LA RÉPUBLIQUE D'HAÏTI. MINISTĖRE DE LA PLANIFICATION ET DE LA COOPÉRATION EXTERNE. 2012. Pays émergent en 2030. 285p. Disponible desde Internet en: http://www.ht.undp.org/content/dam/haiti/docs/ Gouvernance\%20d\%C3\%A9mocratique\%20 et $\% 20$ etat $\% 20$ de $\% 20 \mathrm{droit} /$ UNDP HT PLAN\%20STRAT\%C3\%89GIQUE\%20 de \% $2 \overline{0}$ developpement\%20Haiti_tome1.pdf (con acceso 24/04/18).

31. GOVERNMENT OF BELIZE, MINISTRY OF ECONOMY DEVELOPMENT. 2016. Growth and Sustainable Development Strategy2016-2019. 108p. Available at: https://observatorioplanificacion.cepal.org/sites/ default/files/plan/files/BelizeGSDS.pdf (con acceso 25/05/18).

32. GOVERNMENT OF ST. VINCENT AND THE GRENADINES. CENTRAL PLANNING DIVISION.
MINISTRY OF FINANCE AND ECONOMIC PLANNING. 2013. Plan 2013-2025. 5:53-146. Available at: https://observatorioplanificacion.cepal. org/sites/default/files/plan/files/Saint\%20Vincent\%20 National\%20 conomic $\% 20$ and $\% 20$ social $\% 20$ development\%20plan\%202013\%20-\%202025\%20 partII.pdf (con acceso 13/05/18).

33. GOVERNMENT OF THE REPUBLIC OF SURINAME. 2017. Policy Development Plan 2017-2021. 165p. Available at: https://observatorioplanificacion.cepal. org/sites/default/files/plan/files/SurinamePolicyDevelopment-Plan2017-2021-Partl.pdf (con acceso 03/05/18).

34. GOVERNMENT OF THE REPUBLIC OF TRINIDAD AND TOBAGO. 2016. National Development Strategy 2016-2030 140p. Available at: https://www.planning. gov.tt/sites/default/files/Vision\%202030-20The\%20 National\%20Development\%20Strategy\%20of\%20 Trinidad\%20and\%20Tobago\%202016-2030.pdf (con acceso 27/04/18).

35. GOVERNO FEDERAL BRASIL. MINISTÉRIO DO PLANEJAMENTO, ORÇAMENTO E GESTÃO. 2016. Plano Plurianual 2016-2019. 203p. Disponible desde Internet en: https://observatorioplanificacion. cepal.org/sites/default/files/plan/files/ BrasilPlanPlurianual2016-2019.pdf (con acceso 13/05/18).

36. HERZ, S.; LA VINA, A.; SOHN, J. 2007. Development Without Conflict: The Business Case for Community Consent. World Resources Institute, Washington. (EEUU). 61p.

37. HUMPHEYS, D. 2000. A business perspective on community relations in mining. (Netherlands). Resources Policy 26(3):127-131. https://doi.org/10.1016/ S0301-4207(00)00024-6

38. INTERNATIONAL DESIGN AND ENTERTAINMENT ASSOCIATES INCORPORATED. 2008. Saint Lucia National Vision Plan. 2008. 76p. Available at: https:// www.finance.gov.lc/resources/download/45/.pdf (con acceso 14/05/18).

39. JENKINS, H.; YAKOVLEVA, N. 2006. Corporate social responsibility in the mining industry: Exploring trends in social and environmental disclosure. (Netherlands). J. Cleaner Production 14(4):271-284. https://doi.org/10.1016/j.jclepro.2004.10.004 
40. LEFF, E. 2009. Racionalidad ambiental. La reapropiación social de la naturaleza. Siglo XXI Editores, segunda edición. (México). 509p.

41. MACRI, M. 2015. Objetivos de Gobierno de la Argentina. 12p. Disponible desde Internet en: https:// observatorioplanificacion.cepal.org/sites/default/ files/plan/files/ArgentinaObjetivosdeGobierno.pdf (con acceso 25/04/18).

42. MADURO M., N. 2013. Segundo Plan Socialista de Desarrollo Económico y Social de la Nación 2013-2019, (Venezuela). 148p. Disponible desde Internet en: https://observatorioplanificacion. cepal.org/sites/default/files/plan/files/ VenezuelaPlandelaPatria20132019.pdf (con acceso 04/05/18).

43. MANHATTAN MINERALS CORP. 2004. (1004701) SEC Filing 20-F Annual report for the fiscal year ending Wednesday, December 31, 2003.

44. MARTINEZ-ALIER, J. 2006. Los conflictos ecológico-distributivos y los indicadores de sustentabilidad. Polis. Revista Universidad Bolivariana de Chile. 5(13):1-12.

45. MINISTERIO DE ECONOMÍA, PLANIFICACIÓN Y DESARROLLO. CONSEJO NACIONAL DE REFORMA DEL ESTADO. 2010. Estrategia Nacional de Desarrollo 2010-20130. (República Dominicana). 153p. Disponible desde Internet en: https:// observatorioplanificacion.cepal.org/sites/default/ files/plan/files/RepublicaDominicanaEstrategia NacionaldeDesarrollo.pdf (con acceso 08/05/18).

46. MINISTRIES OF GOVERNMENT OF ANTIGUA AND BARBUDA. 2016. Medium Term Strategic Development Plan. 621p. Available at: https://ab.gov.ag/pdf/ budget/2016_Budget_Summary.pdf (con acceso 03/05/18).

47. MINISTRY OF THE PRESIDENCY. 2017. Green State Development Strategy of Guyana. 62p. Available at: http://www.greengrowthknowledge.org/sites/default/ files/Framework\%20for\%20Guyana\%20Green\%20 State\%20Development\%20Strategy\%2028-03-17. pdf (con acceso 24/04/18).

48. OBSERVATORIO DE CONFLICTOS MINEROS DE AMÉRICA LATINA -OCMAL-. 2015. Auge de minería en Latinoamérica. Informe de referencia. (Chile). $12 \mathrm{p}$.

49. OBSERVATORIO DE CONFLICTOS MINEROS DE
AMÉRICA LATINA -OCMAL-. 2018. Conflictos Mineros en América Latina. Disponible desde Internet en: https://mapa.conflictosmineros.net/ocmal_dbv2/ (con acceso 15/05/18).

50. PINEDO, C. 2014. Anglo American ratifica que en el 2015 desarrollará el proyecto Quellaveco. La República. Disponible desde Internet en: https://larepublica. pe/archivo/773351-anglo-american-ratifica-que-el2015-desarrollara-el-proyecto-minero-quellaveco (con acceso 01/04/18).

51. PIÑERA, S. 2017. Programa de Gobierno 20182022, Construyamos tiempos mejores para Chile. 193p. Disponible desde Internet en: https://observatorioplanificacion. cepal.org/sites/default/files/plan/files/ chileprogramadegobiernodesebastianpinera.pdf (con acceso 03/05/18).

52. PLANNING INSTITUTE OF JAMAICA. 2009. Vision 2030. 376p. Available at: https://sustainabledevelopment. un.org/content/documents/1501jamaica.pdf (con acceso 15/04/18).

53. PRESIDENCIA, OFICINA DE PLANEAMIENTO Y PRESUPUESTO. 2017. Hacia una Estrategia Nacional de Desarrollo Uruguay 2050. 24p. Disponible desde Internet en: https://www.opp.gub.uy/images/ Hacia_una_Estrategia_Nacional_de_Desarrollo_ Uruguay_2050.pdf (con acceso 04/05/18).

54. REPÚBLICA DE COLOMBIA. DEPARTAMENTO NACIONAL DE PLANECIÓN. 2015. Plan Nacional de Desarrollo 2014-2018. 783p. Disponible desde Internet en https://www.dnp.gov.co/Plan-Nacionalde-Desarrollo/paginas/que-es-el-plan-nacional-dedesarrollo.aspx (con acceso 15/04/18).

55. REPÚBLICA DE HONDURAS. 2010. Visión de País 2010-2038 y Plan de Nación 2010-2022. 177p. Disponible desde Internet en https:// observatorioplanificacion.cepal.org/sites/default/ files/plan/files/HondurasPlandeNacion20102022.pdf (con acceso 15/04/18).

56. RODRÍGUEZ, I.; CORREA, H.D. 2003. Lecciones, enfoques y retos en la transformación de conflictos socioambientales en américa latina la experiencia del programa "CyC". (Costa Rica). Encrucijadas Ambientales en América Latina: p.23-102.

57. SECRETARÍA NACIONAL DE PLANIFICACIÓN Y DESARROLLO. 2017. Plan Nacional Para el Buen Vivir 
2017-2021. (Ecuador). 159p. Disponible desde Internet en: https://observatorioplanificacion.cepal.org/ sites/default/files/plan/files/EcuadorPlanNacionalparaelBuenVivir2017-2021.pdf (con acceso 10/04/18).

58. SECRETARÍA TÉCNICA Y DE PLANIFICACIÓN GOBIERNO DE EL SALVADOR. 2015. Plan quinquenal de desarrollo 2014-2019. 270p. Disponible desde Internet en: https://observatorioplanificacion. cepal.org/sites/default/files/plan/files/ELSAL.pdf (con acceso 16/04/18).

59. S\&P GLOBAL. 2017. Worldwide Mining Exploration Trends. (EEUU). 15p. Available at: http:// mineralsmakelife.org/wp-content/uploads/2017/04/ Worldwide_Mining_Exploration_Trends_2017.pdf (con acceso 10/04/18).

60. TWEREFOU, D.K. 2009. Mineral Exploitation, Environmental Sustainability and Sustainable Development in EAC, SADC and ECOWAS Regions. (Ethiopia). African Trade Policy Centre (79). 43p.

61. UNITED NATIONS. 2002. Report of the World Summit on Sustainable Development Johannesburg, South Africa. (EEUU). 167p. Available at: http:// www.un.org/ga/search/view_doc.asp?symbol=A/ CONF.199/20\&Lang=E (con acceso 25/04/18).

62. UNITED NATIONS. 2012. Toolkit and guidance for preventing and managing land and natural resources conflict. Conflict prevention in resource-rich economies. (EEUU). 48p.

63. UNIVERSITY OF EASTERN OF FINLAND. 2012. Responsible Mining: A toolkit for the prevention and mediation of conflicts in the development of the mining sector. 60p. Disponible en: http://epublications.uef. fi/pub/urn_isbn_978-952-61-0926-8/urn_isbn_978952-61-0926-8.pdf (con acceso 20/05/18).

64. VIANA, R. 1997. Bases para el ordenamiento ambiental territorial del municipio de La Peña Cundinamarca. INGEOMINAS. (Colombia). 75p. Disponible desde Internet en: https:// miig.sgc.gov.co/Paginas/Registrolnformacion. aspx? Licencia $=1 \&$ Descarga $=1 \&$ IDElemnent $=$ $87647 \& U$ rlDescarg=http://recordcenter. sgc.gov.co/B 5/13012002520297/ documento/pdf/0101202971102000. pdf\&Tit $=$ Ordenamiento $\% 20$ ambiental $\% 20$ territorial\%20 en $\% 20$ el\%20municipio\%20 de\%20la\%20Pena,\%20Cundinamarca\%20 - \% 20 Propuesta\% 20 tecnico\% 20 - \% 20 Economica.\&RutaRedireccionada =https:// miig.sgc.gov.co/Paginas/ResultadosHijos. aspx?PadreMiig $=\% 221301201010025202970000$ 00000\%22\&k=la\%20pe\%C3\%B1a (con acceso 28/05/18).

65. VIANA R., R.; VELÁSQUEZ M., E.; PEREZ C., R. 1999. Evaluación del potencial ambiental de los recursos suelo, agua, mineral y bosques en el territorio de jurisdicción de Cardique. INGEOMINAS CARDIQUE. (Colombia). 284p. Disponible desde Internet en: http://www.iaea.org/inis/collection/ NCLCollectionStore/_Public/36/036/36036643.pdf (con acceso 28/05/18).

66. VIANA, R.; SOTO M., J.M.; GONZÁLEZ, M.; PONCE, A.; PINTO, E.; CABRERA, M.; NEGRETE, R.; VEGA, A. 2005. Incorporación de la Actividad Minera en los Procesos de Ordenamiento Territorial. Serie Ambiente y Ordenamiento Territorial (2). Ministerio de Ambiente, Vivienda y Desarrollo Territorial. (Colombia). 64p. Disponible desde Internet en: http:// www.cridlac.org/digitalizacion/pdf/spa/doc17084/ doc17084-contenido.pdf (con acceso 29/05/18).

67. VISCIDI, L.; FARGO, J. 2015. Local conflicts and natural resources A balancing act for Latin American Governments. THEDIALOGUE, Energy Working paper. (EEUU). 12p.

Recibido: Julio 15 de 2018

Aceptado: Noviembre 1 de 2018

Cómo citar:

Viana Ríos, R. 2018. Minería en América Latina y el Caribe, un enfoque socioambiental. Rev. U.D.C.A Act. \& Div. Cient. 21(2):617-637. https://doi.org/10.31910/rudca.v21.n2.2018.1066 